\title{
Modification of the tumour microenvironment via exosomal shedding of sphingosine 1-phosphate receptor 2 by breast cancer cells
}

\author{
Stuart M. Pitson and Jason A. Powell \\ News on: The sphingosine 1-phosphate receptor 2 is shed in exosomes from breast cancer cells and is $\mathrm{N}$-terminally processed to a \\ short constitutively active form that promotes extracellular signal regulated kinase activation and DNA synthesis in fibroblasts by El \\ Buri et al. Oncotarget. 2018; 9:29453-29467. https://doi.org/10.18632/oncotarget.25658
}

Sphingosine-1 phosphate (S1P), formed by the phosphorylation of sphingosine by the sphingosine kinases, is a bioactive signaling lipid that can promote cell proliferation, survival, differentiation and migration [1]. S1P has been widely implicated in cancer initiation, progression and chemotherapeutic resistance via its actions as a ligand for a family of five S1P-specific G protein-coupled receptors (GPCRs), named $\mathrm{S}_{1} \mathrm{P}_{1}$ to $\mathrm{S}_{\mathrm{P}}$, and as an intracellular second messenger that can alter gene transcription [2]. S1P can engage its GPCRs in both an autocrine and paracrine fashion to promote classical GPCR signaling cascades and biological responses. Specifically $\mathrm{S}_{1} \mathrm{P}_{2}$ is located on the plasma membrane and coupled to $\mathrm{G}_{\mathrm{i}}, \mathrm{G}_{\mathrm{q}}$ and $\mathrm{G}_{12 / 13}$, and upon S1P binding activates phospholipase $\mathrm{C}$, Rho and ERK1/2 signaling pathways. Despite S1P being classically involved in tumorigenic signaling, the role of $\mathrm{S}_{1} \mathrm{P}_{2}$ in this process has been ambiguous with different studies indicating that this receptor can either promote [3-5] or inhibit [6] tumorigenesis.

El Buri et al. [7] in this issue of Oncotarget have identified a new novel mechanism by which $\mathrm{S}_{1} \mathrm{P}_{2}$ regulates the tumour microenvironment. The authors hypothesize that, like the sphingosine kinases [8], the S1P receptors might also be shed from breast cancer cells in exosomes to regulate the tumour microenvironment. Initial experiments indeed showed that $\mathrm{S}_{1} \mathrm{P}_{2}$ was released into the conditioned medium from MDA-MB-231 breast cancer cells, which induced robust ERK1/2 activation and DNA synthesis/ cell proliferation of mouse embryonic fibroblasts (MEFs). Using a combination of exosome isolation, western blotting, immunofluorescence and electron microscopy the authors clearly demonstrated the $\mathrm{S}_{1} \mathrm{P}_{2}$ was derived from exosomes shed from breast cancer cells and taken up by the MEFs. To exclude the possibility that additional cargo within the exosomes contribute to the activation of ERK-1/2 signaling cascade in fibroblasts, the authors knocked down $\mathrm{S}_{1} \mathrm{P}_{2}$ in MDA-MB-231 cells and showed exosomes from scrambled but not $\mathrm{S}_{1} \mathrm{P}_{2}$ siRNA treated MDA-MB-231 cells promoted ERK-1/2 activation. Unexpectedly, however, the ERK $1 / 2$ activation by this conditioned medium was insensitive to the $\mathrm{S} 1 \mathrm{P}_{2}$ receptor antagonist JTE-013, implying that the $\mathrm{S}_{1} \mathrm{P}_{2}$ released from breast cancer cell-derived exosomes is no longer able to bind JTE-013. Indeed, the evidence suggested that this exosomal $\mathrm{S}_{1} \mathrm{P}_{2}$ is taken up by fibroblasts and processed at the N-terminus to a smaller ( $36 \mathrm{kDa}$ versue the normal 40 $\mathrm{kDa}$ protein) constitutively active form. This $\mathrm{N}$-terminal truncated form of $\mathrm{S}_{1} \mathrm{P}_{2}$ induced significant morphological rounding of MEFs providing further evidence that this form of $\mathrm{S}_{2} \mathrm{P}_{2}$ is biologically active. Interestingly, the authors also identified a similarly truncated species of $\mathrm{S}_{1} \mathrm{P}_{2}$ in the MEFs. The appearance of this endogenous MEF-derived truncated $\mathrm{S}_{2} \mathrm{P}_{2}$, however, was blocked by JTE-013 suggesting that, unlike the exosomal-derived $\mathrm{S}_{1} \mathrm{P}_{2}$, it may be processed in response to engagement of S1P.

The identification of the putative protease present in MEFs that cleaves the N-terminus of exosomal S1P will be of great interest to the field. To this end El Buri et al. [7] identified a putative intramembrane metalloprotease cleavage site near the N-terminus of $\mathrm{S}_{1} \mathrm{P}_{2}$. While it remains to be determined if this is the physiologically relevant cleavage site, notably, a $\mathrm{S}_{1} \mathrm{P}_{2}$ mutant truncated to this residue phenocopied the exosomal-derived truncated $\mathrm{S}_{1} \mathrm{P}_{2}$ in activating ERK-1/2 signaling. El Buri et al. have also begun to address the mechanism by which a truncated $\mathrm{S}_{1} \mathrm{P}_{2}$ induces ligand independent activation of ERK-1/2 signaling. Using the crystal structures of the homologous $\mathrm{S}_{1} \mathrm{P}_{1}$ receptor they generated a structural model of $\mathrm{S}_{1} \mathrm{P}_{2}$. From this they hypothesized that in the inactive state the $\mathrm{N}$-terminus of $\mathrm{S}_{1} \mathrm{P}_{2}$ may tension its transmembrane helix (TM)1 to maintain a compressive force on TM7. This potentially results in stabilization of the interface between the intracellular ends of TM7 and TM6 constraining S1P signaling. Removal of the N-terminal peptide is predicted to relax TM1 resulting in separation of TM6 and TM7 permitting $G$ protein engagement and constitutive activity of the receptor.

These studies raise a number of critical questions that should be addressed in the future. Firstly, what are the consequences of exosomal $\mathrm{S}_{1} \mathrm{P}_{2}$ for the tumor microenvironment in vivo, and does it contribute to a microenvironment conducive to metastatic spread? 
Secondly, what regulates the release of exosomal $\mathrm{S}_{1} \mathrm{P}_{2}$ from breast cancer cells, and does this occur in other cancers? Thirdly, what is the exact cleavage site and the accompanying protease that processes exosomal $\mathrm{S}_{1} \mathrm{P}_{2}$. Answers to these questions may uncover novel therapeutic targets to manipulate the supportive tumour microenvironment.

These findings of El Buri et al. [7] are important as this is the first demonstration of exosomal-derived $\mathrm{S}_{1} \mathrm{P}_{2}$ which has the potential to regulate the tumour microenvironment. In addition to breast cancer, this may have broad reaching implications to other cancers that have a dependence on $\mathrm{S}_{1} \mathrm{P}_{2}$ and provide novel avenues for therapeutic development.

Stuart M. Pitson: Centre for Cancer Biology, University of South Australia and SA Pathology, North Tce, Adelaide SA, Australia

Correspondence to: Stuart M. Pitson, email stuart.pitson@unisa.edu.au

Keywords: sphingosine 1-phosphate; exosomes; breast cancer; tumour microenvironment

Received: June 18, 2018

Published: July 24, 2018

\section{REFERENCES}

1. Pyne et al. Nat Rev Cancer. 2010; 10:489-503.

2. Parham et al. FASEB J. 2015; 29:3638-3653

3. Powell et al. Blood. 2017; 129:771-782

4. Salas et al. Blood. 2011; 117:5941-5952

5. Ponnusamy et al. EMBO Mol. Med. 2012; 4:761-775

6. Sugimoto et al. Mol Cell Biol. 2003; 23:1534-1545

7. El Buri et al. Oncotarget. 2018; 9:29453-29467. https://doi.org/10.18632/oncotarget.25658

8. Rigogliuso et al. J Oncol. 2010; 201:509329

Copyright: Pitson et al. This is an open-access article distributed under the terms of the Creative Commons Attribution License 3.0 (CC BY 3.0), which permits unrestricted use, distribution, and reproduction in any medium, provided the original author and source are credited. 